\title{
Evaluation of anticoagulant control in a pharmacist operated anticoagulant clinic
}

\author{
A S Radley, J Hall, M Farrow, P J Carey
}

\begin{abstract}
Aims-To compare the quality of outpatient anticoagulant control before and after the transfer of dosing responsibility to designated trained pharmacists from rotating junior medical staff.

Methods-All International Normalised Ratio (INR) values for an eight month period either side of the staff changeover were assessed for precision of therapeutic control according to described standards. Allowing for patient associated effects, observed and expected frequencies of "successful" control for the two staff groups were compared under the hypothesis of no association.
\end{abstract}

Results-INR results $(n=2219)$ for 382 patients were analysed. For patients in stable therapeutic control, there was no significant difference in performance between the two staff groups. Patients with an INR result "out" of control limits were more likely to be returned "in" to control at their next visit by the pharmacists than by the doctors.

Conclusions-The quality of anticoagulant control in outpatient clinics benefits from dedicated trained staff using standard protocols.

( $f$ Clin Pathol 1995;48:545-547)

Keywords: Pharmacists, anticoagulants.

Oral anticoagulants are a standard therapy in the treatment of thromboembolic disease. Indications for their use have expanded and increasing numbers of patients now take these drugs. ${ }^{1}$ Many require lifelong treatment and are regularly monitored in hospital outpatient clinics. The optimal level of anticoagulation depends upon the underlying condition and guidelines have been proposed by the British Society for Haematology. ${ }^{2}$ The control of anticoagulation is influenced by many factors including intercurrent disease and drug therapy, and knowledge of these aids accurate control.

Institutions vary in the type of staff who undertake dosage control in anticoagulant clinics. Examples include consultant haematologists, clinical assistant medical staff, rotating junior medical staff, and pharmacists. Computer programs are sometimes used to assist dosage recommendations.

Involvement in anticoagulant therapy is an accepted part of pharmacy practice. Pharmacist run anticoagulant clinics have been closely evaluated in the United States and have been shown to offer a high standard of patient care and an effective use of resources. ${ }^{45}$ Pharmacist run clinics have been in operation in Great Britain since $1979^{6}$ and about 14 clinics are currently established. A clinic managed by pharmacists has operated within Sunderland Hospitals since January 1991 using standard protocols and guidelines developed jointly by physicians and pharmacists and accepted by the Health $\mathrm{Au}$ thority.

In the pharmacist clinic at Sunderland documentation has been developed for each patient which defines the indication for anticoagulation, the intensity of anticoagulation and length of treatment required. A full drug history is maintained and factors that might be expected to affect therapeutic control are noted. At each clinic visit, the patient is interviewed by the pharmacist who titrates anticoagulant dosage according to the International Normalised Ratio (INR).

The opportunity to evaluate the impact of a pharmacist operated clinic on the quality of anticoagulant control was presented when the staffing of the anticoagulant clinic at Sunderland Royal Infirmary changed from junior medical staff. This study compares the performance of the two clinic arrangements in the ability to maintain therapeutic INR control.

\section{Methods}

DATA COLLECTION

The anticoagulant records of all patients attending the outpatient anticoagulant clinic at Sunderland Royal Infirmary between January and September 1992 were studied retrospectively. During each clinic visit, the patients' INR values were determined by a haematology technician. The laboratory methods, reagent type and instrumentation did not change during the study period. Before April 1992, dosage was regulated at the discretion of the clinic doctor who was one of a pool of rotating medical senior house officers or registrars. From April 1992, the operation of the clinics was undertaken by pharmacist members of the anticoagulant team.

The quality of anticoagulant control was analysed using audit standards developed for the anticoagulant service at Sunderland Hospitals. ${ }^{7}$ The local standards define an acceptable precision of control as compliance with the prescribed INR range plus or minus $0 \cdot 5$. INR values recorded in this way were analysed by means of a database facility at the University of Sunderland (dBase IV, Ashton Tate).

\section{STATISTICAL ANALYSIS}

To avoid the need for a more complex statistical model, involving assumptions about the forms 
Table 1 The proportion of patients seen by each clinic

\begin{tabular}{lrr}
\hline Both clinics & 261 & $68 \cdot 3 \%$ \\
Doctor clinic & 71 & $18 \cdot 6 \%$ \\
Pharmacist clinic & 50 & $13 \cdot 1 \%$ \\
\hline
\end{tabular}

Table 2 The diagnosis of patients requiring anticoagulation

\begin{tabular}{lrl}
\hline Principle diagnosis & Number & Mean duration of $R_{\mathrm{x}}$ \\
\hline Valve prosthesis & 92 & \\
Valve disease/AF & 127 & \\
Cardiomyopathy AF & 10 & \\
CABG & 5 & \\
AF and emboli & 32 & Long term \\
Lone AF & 19 & \\
Carotid disease & 3 & \\
Peripheral arterial disease & 29 & \\
Recurrent DVT & 5 & \\
Lupus anticoagulant/stroke & 2 & \\
DVT & 33 & 3 months \\
Pulmonary embolism & 25 & 6 months \\
Total & 382 & \\
\hline
\end{tabular}

$\mathrm{AF}=$ atrial fibrillation; $\mathrm{CABG}=$ coronary bypass grafting; DVT $=$ deep vein thrombosis.

of probability distributions, the observations were classified as belonging to one or other of the two states "in" or "out" of the prescribed range plus or minus $0 \cdot 5$. This permitted a nonparametric comparison of the proportions "in" achieved by the physicians and pharmacists.

It seemed likely that the probability of successful control-that is, achieving the state "in", would vary between patients and also according to each patient's recent history. Individual patients were observed several times during the course of the study. Some patients were seen only by the physicians, some only by the pharmacists and some were seen before and after the change in clinic management. There was no randomisation or matching of patients between physicians and pharmacists. For these reasons, it was necessary to allow for possible within-patient dependence in the data. As an initial analysis, McNemar's test ${ }^{8}$ was used to compare results between the last two physician visits and the first two pharmacist visits, for those patients where these data were available. This avoids the possibility of dominance of the results by unstable patients who might contribute more data and also to reduce any time effects.

This test was supplemented by an analysis of the whole data set. With such a large number of patients and a small number of observations per patient, the fitting of explicit patient effects was rejected in favour of a Markov chain model. In this model the probability of successful control on one occasion depends on the state of that patient at the previous occasion but, given that previous state, there is no further de-
Table 4 INR status at observed clinic visit for each previous INR state

\begin{tabular}{llll}
\hline & Doctors & Pharmacists & Total \\
\hline $\begin{array}{l}\text { Previously out } \\
\text { out }\end{array}$ & $\begin{array}{ll}130 \\
(113 \cdot 54)\end{array}$ & $\begin{array}{l}71 \\
(87 \cdot 46)\end{array}$ & 201 \\
in & $\begin{array}{l}153 \\
(169 \cdot 46)\end{array}$ & $\begin{array}{l}147 \\
(130 \cdot 54)\end{array}$ & 300 \\
total & 283 & 218 & 501 \\
$\chi_{1}^{2}=9 \cdot 159$ & & & \\
$\begin{array}{l}\text { Previously in } \\
\text { out }\end{array}$ & 146 & 128 & 274 \\
in & $(146 \cdot 20)$ & $(127 \cdot 80)$ & \\
total & 569 & 497 & 1066 \\
$\chi_{1}^{2}=0 \cdot 001$ & $(568 \cdot 80)$ & $(497 \cdot 20)$ & 1340 \\
Previously unknown & 715 & 625 & \\
out & & & \\
in & 93 & 12 & 105 \\
total & $(91 \cdot 67)$ & $(13 \cdot 33)$ & \\
$\chi_{1}^{2}=0 \cdot 211$ & 237 & 36 & 273 \\
\hline
\end{tabular}

pendence on earlier history. (A third state, "unknown", was assigned for the previous occasion when the INR was the first listed for the patient.)

\section{Results}

The values of 2219 INRs for 382 patients were analysed. Table 1 shows the number and proportion of patients in the study who were managed by the doctor clinic only, by the pharmacist clinic only or were involved throughout the changeover. Table 2 gives a breakdown of the indications for anticoagulation. The clinic used a treatment range of 3.0 to 4.0 for therapy of patients with valve prosthesis and a treatment range of 2.0 to 3.0 for other indications. For the purposes of audit and control, local standards defined the degree of precision of control as the prescribed INR range plus or minus 0.5 INR units. Thus, patients with valve prosthesis were, in effect, maintained within a range of 2.5 to 4.5 and patients with other diagnoses within a range of 1.5 to 3.5 .

The numbers "in" at the last two physician occasions and the first two pharmacist occasions were $179,178,184$, and 179 , respectively, of 231 cases considered, showing little change. Fifty five patients did better with pharmacists while 51 did worse, leading to a non-significant result in McNemar's test of the hypothesis of no change in mean overall proportions, based only on these four occasions.

Table 3 shows observed frequencies with expected frequencies, under the hypothesis of no association, in parenthesis. These indicate that there are differences among the success probabilities, when the data are grouped in this

Table 3 INR status of patients at observed clinic visit compared with previous visit

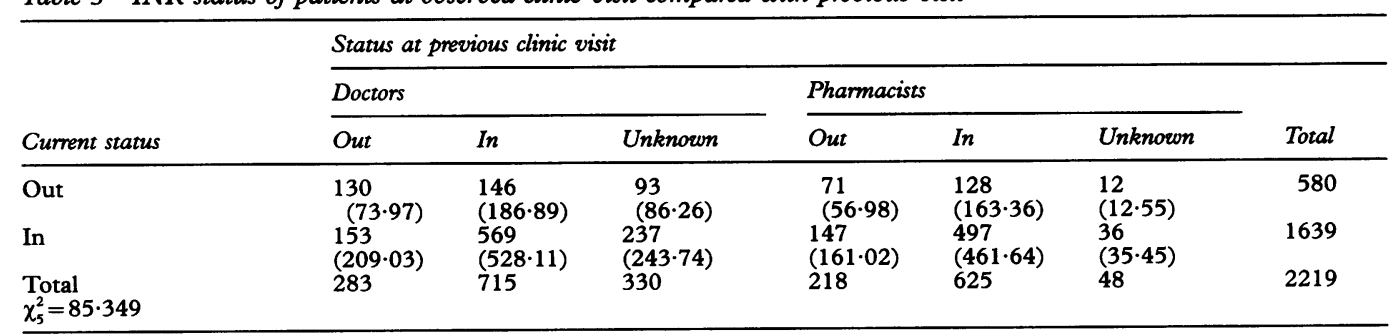


way $(p<0.001)$. Further analysis was undertaken to ascertain whether it was necessary to allow for the previous state and also whether there was evidence of a difference between the pharmacists and the physicians in their control of patients. Contingency tables separated by the previous state and their $\chi^{2}$ tests are presented in table 4.

\section{Statistical calculation}

From results in table 4 , adding together $\chi^{2}$ values:

$$
9 \cdot 159+0 \cdot 001+0 \cdot 211=9 \cdot 371 \text { on } 3 \text { Df }
$$

subtracting this from the $85 \cdot 349$ in table 3 gives 75.978 on $2 \mathrm{Df}$ indicating that it is necessary to allow for the previous state $(p<0.001)$. Looking at the three previous states separately (table 4 ); when the previous state is either "in" or "unknown", there is no evidence of a difference $\left(\chi^{2}=0.001\right.$ and $\chi^{2}=0.211$, respectively) between the doctor and pharmacist data sets. However, if the observation from the previous visit was "out", a value of $\chi^{2}=9 \cdot 159$ is obtained showing a significant difference $(0.001<$ $\mathrm{p}<0.01)$. It appears, therefore, that under the new clinic arrangements a greater number of previously "out" results return to range.

\section{Discussion}

The recent audit of anticoagulant treatment published on behalf of the British Society for Haematology ${ }^{10}$ has highlighted a lack of precision in therapeutic control in anticoagulated patients, a lack of standardisation in therapeutic standards and has recommended an increase in educational input by consultant haematologists. Other authors have criticised the quality of anticoagulant control in hospital outpatient clinics. ${ }^{11-13}$ Junior doctor involvement in these clinics has been questioned through lack of continuity, training, knowledge, and commitment due to more pressing duties. ${ }^{14}$

A similar situation existed in the United States where pharmacist operated clinics have reported improved control of anticoagulant therapy. ${ }^{1516}$ These authors suggested that their results were achieved through continuity of care. The turnover of clinic personnel in their pharmacist clinics was low when contrasted with the previous situation of high junior medical staff turnover. The pharmacist clinics were operated within established guidelines to standardise the general aspects of patient management and each pharmacist underwent specialist training and assessment before taking responsibility for patient care within the clinic. This training included a grounding in the pharmacokinetic principles of warfarin dosing. In this environment direct patient contact and a patient-provider relationship was established and improved patient awareness of anticoagulant therapy through counselling and education was encouraged.
When embarking on a service development, it is important to ensure that patient care does not deteriorate through the change. Maintenance of good patient care in the clinic in Sunderland has been enabled by close cooperation between disciplines and the use of protocols and recognised standards. This has created an environment in which pharmacists' skills in dosage titration, patient counselling and management of adverse drug reactions could best be utilised. The relatively new tool of professional audit has helped clinic personnel to focus on the aims of improved anticoagulant control and reduced thromboembolic and haemorrhagic complications. ${ }^{17}$

The results of the comparison of control reported in this study demonstrate the benefits of dedicated staffing, specialised training, standard protocols, and defined treatment objectives for each patient. With the increasing pressure on health authorities to reduce junior doctors' hours and the increasing number of patients requiring anticoagulation, it would seem that pharmacist managed clinics offer a suitable alternative for managing the care of patients on anticoagulant drugs. The system used in Sunderland appears to overcome some of the problems described in the recent $\mathrm{BCSH}$ audit of anticoagulant treatment and we would advocate its use in other clinics.

1 Nolan J, Bloomfield P. Non-rheumatic atrial fibrillation Warfarin or aspirin for all? Br Heart $\mathcal{F}$ 1992;68:544-8.

2 British Society for Haematology. Guidelines on oral anticoagulation: second edition. f Clin Pathol 1990;43:177-83.

3 Carter BL. Therapy of acute thromboembolism with heparin and warfarin. Clin Pharm 1991;10:501-18.

4 Conte RR, Kehoe WA, Nielson N, Lodhia H. Nine year experience with a pharmacist-managed anticoagulation clinic. Am f Hosp Pharm 1986;43:2460-4.

5 Gray DR, Garabedian-Ruffalo SM, Chretian SD. Costjustification of a clinical pharmacist-managed anticoagulant clinic. Drug Intell Clin Pharm 1985;19:575-80.

6 Pegg M, Bourne JG, Mackay AD, Lawton WA, Cole RB The role of the pharmacist in the anticoagulant clinic. $\dot{f}$ $R$ Coll Physicians Lond 1985;19:39-45.

7 Radley AS, Hall J. Establishment and evaluation of a pharmacist-managed anticoagulant clinic. Pharm $\mathcal{F}$ 1994;252: 91-2.

8 Cox DR, Snell EJ. Binary time series. In: Analysis of binary data. 2nd edn. London: Chapman and Hall, 1989:98-100.

9 Crowder MJ, Hand DJ. Crossover trials with a binary response. In: Analysis of repeated measures. London: Chapman and Hall, 1990:109.

10 Rose PE. On behalf of the BCSH Haemostasis and Thrombosis Task Force of the British Society for Haematology. Audit of oral anticoagulant treatment. F Clin Pathol 1993 46:1069-70.

11 McInnes GT, Helenglass G. The performance of clinics for outpatient control of anticoagulation. $\mathcal{F} R$ Coll Physicians Lond 1987;21:42-5.

12 Harris AD, Birtwell AJ, Jones DB. Anticoagulant control. Lancet 1981;i:1320.

13 Pell JP, McIver B, Stuart P, Malone DN, Alcock J. Comparison of anticoagulant control among patients attending general practice and a hospital anticoagulant clinic. $\mathrm{Br} \mathcal{F}$ Gen Pract 1993;43:152-4.

14 Duxbury BMCD. Therapeutic control of anticoagulant treatment. BMf 1982;284:702-4.

15 Reinders TP, Steinke WE. Pharmacist management of anticoagulant therapy in ambulant patients. Am $\mathcal{F}$ Hosp Pharm 1979;36:645-8.

16 Ellis RF, Stephens MA, Sharp GB. Evaluation of a pharmacy-managed warfarin-monitoring service to coordinate inpatient and outpatient therapy. Am $\mathcal{F}$ Hosp Pharm 1992; 49:387-94.

17 Audit in pharmacy. Working Party Report of the Royal Pharmaceutical Society Pharmaceutical Journal: 18 April,
1992:505-9. 